\title{
A similarity approach to the atmospheric dynamics of giant extrasolar planets and brown dwarfs
}

\author{
A. Sanchez-Lavega* \\ Dpto. Fisica Aplicada I, Escuela Superior de Ingenieros, Universidad del Pais Vasco, Alameda Urquijo s/n, \\ 48013 Bilbao, Spain
}

Received 12 February 2001 / Accepted 16 July 2001

\begin{abstract}
We present an assessment of the most plausible dynamical regimes operating in the atmospheres of giant extrasolar planets (EGP) and cold ("methane") brown dwarfs from the available data on a selected group of objects. The most important parameters controlling the atmospheric circulation are the rotation angular velocity and the energy balance between the internal heat source and the star's insolation. The first parameter can be reasonably constrained for some of these objects by theoretical arguments. The second is constrained by the observations. Assuming a hydrogen composition, we discuss possible scenarios for the first order atmospheric motions in terms of characteristic geophysical fluid dynamic numbers and representative time constants. The analysis is applied to the family of extrasolar giant planets classified recently by Sudarsky et al. (2000) according to their effective temperature and Bond albedo. For completeness we extend this study to cold ("methane") brown dwarfs. Three main dynamical regimes emerge from this analysis: (A) Close EGP ("hot jupiters") with spin-orbit locked (slowly rotating) planets, have their atmospheres mainly under the star's radiative control. Super-rotating atmospheric motions between the heated and cooled hemispheres can be expected. (B) Atmospheres with their dynamics controlled by both the internal and external energy sources, with Coriolis forces producing zonal motions (Jupiter like objects). (C) Cold brown dwarfs, with motions controlled by the internal heat source (thermally driven turbulent convection) producing intense vertical velocities that dominate the motion field.
\end{abstract}

Key words. planets and satellites: general - stars: low mass, brown dwarfs - planetary systems

\section{Introduction}

The increasing discovery of giant extrasolar planets and cold brown dwarfs and the development of theoretical models of their internal structure has opened the possibility of studying their atmospheric characteristics and comparing them to those of the planets of the solar system. One important aspect of a planetary atmosphere is the dynamical regime of its troposphere since the clouds and the meteorological phenomena develop there, which in turn controls the visual appearance of the planet. Dynamics is a very complicated subject because different processes intervene in a nonlinear manner. Even after a series of insitu spacecraft observations of most of the solar system planets we do not still know, for example, what the origin of the Venus super-rotation is (Gierasch et al. 1999), or that of the multiple zonal jets observed on Jupiter and Saturn (Ingersoll 1990). However, a first order approach to atmospheric circulation and meteorology can be derived from the values and scale ratios of the fundamental magnitudes that control the dynamics. A first attempt at

\footnotetext{
* e-mail: wupsalaa@bi.ehu.es
}

such a goal was performed several years ago for the giant planets by Hide (1966), and more generally for the solar system bodies by Golitsyn $(1979,1984)$, being usually refered to a "similarity theory" (see also Gierash et al. 1970). Application of this theory to the atmospheric dynamics of extrasolar planets and cold brown dwarfs is at this moment somewhat speculative since we lack precise measurements of important parameters. However, some of them are reasonably constrained by the observations and by the atmospheric and internal structure models of these bodies. This allows us to make a first classification scheme of the dynamics on extrasolar planets based on the similarity approach.

Knowledge of the basic aspects of the atmospheric dynamics is important in interpreting future photometric and spectroscopic observations of these bodies. Dynamics allows transport of and mixes compounds, activating the atmospheric chemistry and haze and cloud formation. Cloud formation in turn controls the planet's global brightness or albedo (Marley et al. 1999; Sudarsky et al. 2000). The meteorological activity could form discrete features ("bright and dark isolated spots" at particular wavelengths, as in the giant planets), global regular and 
irregular large-scale cloud patterns (as in Venus and Earth), and organized banded albedo patterns (as in the giant planets). These cloud textures could produce temporal photometric and spectroscopic variations due to the planetary rotation or to the seasonal cycle. Measuring these changes with future instrumentation, may allow us to infer the planet rotation and axis tilt. Finally, a comparison of the dynamical regimes present in those atmospheres with that of the solar system giant planets will be crucial to understand the circulation of gaseous hydrogen atmospheres under a variety of situations.

The application of the similarity theory to the atmospheres of giant extrasolar planets and cold brown dwarfs is the subject of this paper. This represents the simplest attempt to understand what kind of motions we can expect in their tropospheres from a comparative point of view. In addition we extend in this paper the work of Golitsyn $(1979,1984)$ including new parameters relevant to understanding the bulk atmospheric dynamics of a giant planet. Instead, to cover a broad casuistic of the possible ranges of values of the similarity parameters, we apply the theory to a selected group of bodies that we consider representative of the extrasolar planets known at present. The natural classification scheme based on effective temperatures (or basically distances) introduced by Sudarsky et al. (2000) for their albedo study is also adopted here. Application of this theory to other bodies (discovered or to be discovered in the future) is straightforward.

\section{Selected objects: Physical data}

Our first fundamental assumption is that the extrasolar planets are giant gas hydrogen bodies, as suggested by their masses and theoretical formation models (Burrows et al. 1997; Guillot 1999). This was nicely confirmed by the unique transit observations of the planet around HD 209458 B (Charbonneau et al. 2000; Henry et al. 2000a; Brown et al. 2001). The lack of a "surface" as a lower boundary for the atmosphere is, together with composition and presumed existence of an internal heat source, the most important and distinctive characteristic from the atmospheric dynamics perspective between a giant and a terrestrial planet. Jupiter's properties will be taken as the reference in this study.

We have selected the objects to be studied from the updated catalog by Schneider (2001) using the separation from the star as a guide. For objects at similar distances, we have used as selection criteria their different masses and ages, and among them, those that have the best determined properties. For example, among the "hot jupiters", $\tau$ Boo B has a much larger mass, is strongly irradiated and probably has a higher internal heat source than 51 Peg B and HD 209458 B. A similar criteria applies also to more distant planets, for example $v$ And D and $47 \mathrm{UMa} \mathrm{B}$. Table 1 list the basic data for the selected bodies. In this table, $a$ is the semi-major orbit axis in astronomical units (a.u.), $t_{\text {orb }}$ is the orbital period, $i$ is the tilt of the orbital plane from the visual, and $M_{*}, L_{*}, t_{*}$ are the mass, luminosity and age of the parent star. References to these data can be found in Schneider (2001). Table 2 summarizes the physical characteristics of the planets as discussed in detail below.

\subsection{Masses}

All objects have the minimum mass $M \sin i$ measured from the radial velocity curves. Their values in Jovian masses $\left(M_{\mathrm{J}}\right)$ are: $M \sin i=0.46(51 \mathrm{Peg} \mathrm{B}), 4.14(\tau$ Boo B $)$, $2.11(v$ And $\mathrm{C}), 4.29$ ( $v$ And $\mathrm{D}), 2.6$ (47 UMa B), 1.2 $(\epsilon$ Eridani B).

The masses $(M)$ of the EGP are at present bounded by constraining the inclination of the orbital plane $i$. For 51 Peg B it is constrained from the lack of transit detection (Henry et al. 1997), and from the observed spectral rotational velocity of the star and its chromospheric emission (François et al. 1996; Henry et al. 1997; Gonzalez 1998). Note that there is, however, some uncertainty in the rotation period of the star (Henry et al. 2000b). For $\tau$ Boo $\mathrm{B}, i$ is constrained from the upper limit detection of the light reflected off of this giant planet (Charbonneau et al. 1999; Cameron et al. 2000). Transit photometry precisely constraints $i$ for HD 209458 B (Henry et al. 2000a; Charbonneau et al. 2000; Mazeh et al. 2000; Jha et al. 2000; Brown et al. 2001). The mass of $\epsilon$ Eridani B is constrained from the tilt observed in its dust disk and from the assumption that this object lies in the disk plane (Hatzes et al. 2000). The mass of $v$ And D is constrained from Hipparcos astrometry (Mazeh et al. 1999; Han et al. 2000). Using then the retrieved value of $i$ for $v$ And $\mathrm{D}$, we estimate the mass of $v$ And $\mathrm{C}$ assuming it lies in the same orbital plane. The mass of $47 \mathrm{UMa} \mathrm{B}$ is constrained from the star's spectroscopy (Gonzalez 1998) and Hipparcos astrometry (Perryman et al. 1996; Han et al. 2000). The mass of Gl 229 B comes from Leggett et al. (1999).

\subsection{Radius}

The radius $\left(R_{\mathrm{p}}\right)$ of the planet is estimated from the evolution models (Burrows et al. 1997, 2000; Guillot 1999) using the age of the star as determined by different methods (see below). The only exception is the planet HD $209458 \mathrm{~B}$ for which a direct measurement of the radius has been performed during transit observations (Charbonneau et al. 2000; Henry et al. 2000a; Mazeh et al. 2000; Jha et al. 2000; Brown et al. 2001). A constraint of the radius of $\tau$ Boo B from the initial tentative detection of the reflected spectra has been given by Cameron et al. (1999) but later modified from a more detailed analysis (Cameron et al. 2000). In fact, the non-detection of the reflected light from this planet puts an upper limit to its radius (Charbonneau et al. 1999) that is consistent with the "inflating" models by Bodenheimer et al. (2001). Their value in Table 2 is given in terms of the Jovian radius $\left(R_{\mathrm{J}}\right.$, $\left.1 R_{\mathrm{J}}=71400 \mathrm{~km}\right)$. The acceleration of gravity $g$ in the 
Table 1. Selected objects and basic data.

\begin{tabular}{ccccccc}
\hline Object & $a($ a.u. $)$ & $t_{\text {orb }}($ days $)$ & $i(\mathrm{deg})$ & $M_{\star}\left(M_{\mathrm{S}}\right)$ & $L_{\star}\left(L_{\mathrm{S}}\right)$ & $t_{\star}(\mathrm{Gy})$ \\
\hline 51 Peg B & 0.052 & 4.231 & $37-85$ & 1.05 & 1.3 & $6-10$ \\
$\tau$ Boo B & 0.047 & 3.313 & $29-46$ & 1.34 & 3.1 & $1.5-2.0$ \\
HD 209458B & 0.046 & 3.52433 & $86.7 \pm 0.25$ & 1.1 & 1.9 & $5-6$ \\
$v$ And C & 0.82 & 241.2 & $\sim 60$ & 1.28 & 3.4 & $3.3-5.5$ \\
$v$ And D & 2.56 & 1308.5 & $\sim 155$ & 1.28 & 3.4 & \\
47 UMa B & 2.09 & 1084 & $44 \pm 24$ & 1.05 & 1.7 & $6.9-8$ \\
$\varepsilon$ Eri B & 3.3 & 2502 & $46 \pm 17$ & 0.85 & 0.3 & $<1$ \\
G1 229B & $\sim 40$ & 92340 & - & 0.6 & 0.015 & $0.5-1$ \\
Jupiter & 5.2 & 4329 & - & 1 & 1 & 4.5 \\
\hline
\end{tabular}

Table 2. Physical characteristics of the objects.

\begin{tabular}{ccccccc}
\hline Object & $\begin{array}{c}M_{\mathrm{p}} \\
\left(\text { in } M_{\mathrm{J}}\right)\end{array}$ & $\begin{array}{c}R_{\mathrm{p}} \\
\left(\mathrm{in} R_{\mathrm{J}}\right)\end{array}$ & $\begin{array}{c}g \\
\left(\mathrm{~m} \mathrm{~s}^{-2}\right)\end{array}$ & $\begin{array}{c}<\rho> \\
\left(\mathrm{gr} \mathrm{cm}^{-3}\right)\end{array}$ & $\begin{array}{c}t_{\mathrm{rot}(\min )} \\
(\mathrm{hr})\end{array}$ & $\begin{array}{c}t_{\mathrm{syn}}=t_{\text {orb }} \\
(\text { days })\end{array}$ \\
\hline 51 Peg B & $0.49-0.8$ & $1.2-1.4$ & $6-14$ & $0.2-0.6$ & $4-7$ & 4.23 (syn) \\
$\tau$ Boo B & $6-8$ & 1.2 & 121 & 3.5 & 2 & 3.31 (syn) \\
HD 209458B & 0.66 & 1.35 & 8 & 0.30 & 5.3 & 3.52 (syn) \\
$v$ And C & 2.4 & 1.06 & 55 & 2.5 & 2.1 & \\
$v$ And D & $10.1 \pm 4.7$ & 1.1 & $94-230$ & $4-15$ & $0.8-1.6$ & \\
47 UMa B & $3.7 \pm 1.1$ & 1.1 & $56-155$ & $2.5-7.3$ & $1.2-2.1$ & \\
$\varepsilon$ Eri B & $1.2 \pm 0.3$ & 1.1 & 28 & 1.1 & 3 & \\
G1 229B & $25-35$ & 1 & $620-870$ & $31-43$ & $0.5-0.6$ & \\
Jupiter & 1 & 1 & 24.2 & 1.24 & $t_{\text {rot }}=9.9 \mathrm{hr}$ & \\
\hline
\end{tabular}

troposphere and the mean density $\langle\rho\rangle$ of the object are derived from these data.

\subsection{Rotation period}

The next parameter, rotation period, is critical for atmospheric dynamics but unfortunately it has not been measured for any of these bodies. We can only constraint it in the lower limit by the condition that the body is not broken apart by the centrifugal force

$$
t_{\mathrm{rot}}(\min )=\left(\frac{3 \pi}{G<\rho>}\right)^{1 / 2}
$$

being $G$ the gravitational constant. For CEGP, the effect of tidal forces should lock the planet spin to the orbital period if the synchronization period $\left(t_{\mathrm{syn}}\right)$ is lower than the age of the star $\left(t_{*}\right)$ being

$$
t_{\mathrm{syn}}=Q \Omega_{i}\left(\frac{M_{\mathrm{p}}}{G M_{*}^{2}}\right)\left(\frac{a^{6}}{R_{\mathrm{p}}^{3}}\right)
$$

(see Marcy et al. 1997 for details). We take $Q \sim 5 \times 10^{5}$ as estimated for Jupiter and, the planet rotation initial angular velocity, $\Omega_{i}$ as $2 \pi / t_{\text {rot }}$ (min). According to these data and to Eq. (2) we find that CEGP have $t_{\text {syn }} \sim 1-$ $2.6 \times 10^{6}$ years which is $<<t_{*}$. This means that these planets most probably have their spin synchronized to the orbital period, an effect that has important consequences for their atmospheric dynamics. Moreover, there is some observational evidence of rapid rotation in brown dwarfs, with periods ranging from 1.5 to $6 \mathrm{hr}$ (Martin et al. 1997; Tinney \& Reid 1998; Tinney \& Tolley 1999). Thus we could expect Gl 229 B to have a rotation period close to this range of values.

\subsection{Heat sources}

In Table 3 we present the data for the sources of atmospheric heating and their related temperatures. Only the primary energy sources (i.e. the insolation from the star and the internal heat) are considered. For class II of Sudarsky et al. planets, the effects related to the latent heat released during the formation of water clouds could also have an important effect on the atmospheric dynamics (Gierasch 1976). For classes IV and V (CEGP) the energy source coming from the gravitational tides could also be important (Bodenheimer et al. 2001). The incident flux on the atmosphere, $F_{*}=L_{*} /\left(4 \pi a^{2}\right)$ is obtained from the data in Table 1. The albedo $(A)$ is taken from the tables in Sudarsky et al. (2000). It depends on the spectral type of the parent star and the type of cloud cover considered (following microphysics and condensate types). The only direct albedo observation comes from Cameron et al. (2000) that constrain the spherical albedo of $\tau$ Boo B to be $A_{\mathrm{s}}<0.33$ in the visible. The flux absorbed by the atmosphere is then given by

$F_{\mathrm{abs}}=(1-A) F_{*} / 4$

and it is used to retrieve the equilibrium temperature $\left(T_{\text {eq }}\right)$

$T_{\mathrm{eq}}=\left[\frac{\left(1-A_{B}\right) L_{*}}{16 \pi \sigma a^{2}}\right]^{1 / 4}$.

The internal luminosity $\left(L_{\text {int }}\right)$ is obtained from the evolution models of Burrows et al. (1997) using the star age 
Table 3. Heat sources and temperatures.

\begin{tabular}{cccccccc}
\hline Object & $\begin{array}{c}F_{\star} \\
\left(\mathrm{W} \mathrm{m}^{-2}\right)\end{array}$ & $A$ & $\begin{array}{c}F_{\text {abs }} \\
\left(\mathrm{W} \mathrm{m}^{-2}\right)\end{array}$ & $\begin{array}{c}T_{\text {eq }} \\
(\mathrm{K})\end{array}$ & $\begin{array}{c}L_{\text {int }} \\
\left(L_{\mathrm{s}}\right)\end{array}$ & $\begin{array}{c}F_{\text {int }} \\
\left(\mathrm{W} \mathrm{m}^{-2}\right)\end{array}$ & $\begin{array}{c}T_{\text {eff }} \\
(\mathrm{K})\end{array}$ \\
\hline 51 Peg B & $6.6 \times 10^{5}$ & 0.03 & $1.6 \times 10^{5}$ & 1300 & $5 \times 10^{-10}$ & 3 & 1300 \\
$\tau$ Boo B & $1.9 \times 10^{6}$ & 0.03 & $4.6 \times 10^{5}$ & 1690 & $10^{-7}$ & 423 & 1690 \\
HD 209458B & $1.2 \times 10^{6}$ & $0.03-0.55$ & $1-3 \times 10^{5}$ & 1350 & $10^{-9}$ & 3 & 1300 \\
$v$ And C & 6886 & 0.14 & 1480 & 402 & $6 \times 10^{-9}$ & 34 & 404 \\
$v$ And D & 1317 & $0.4-0.8$ & $36-108$ & 184 & $3 \times 10^{-8}$ & 151 & 250 \\
47 UMa B & 542 & $0.4-0.8$ & 90 & 195 & $10^{-8}$ & 55 & 210 \\
$\varepsilon$ Eri B & 38 & 0.45 & 5 & 97 & $8 \times 10^{-9}$ & 40 & 168 \\
G1 229B & 0.013 & - & 0.002 & 14 & $6 \times 10^{-6}$ & 39555 & 900 \\
Jupiter & 51 & 0.343 & 8.1 & 109 & $8.7 \times 10^{-10}$ & 5.4 & 124 \\
\hline
\end{tabular}

given in Table 1 . The internal heat flux $\left(F_{\text {int }}\right)$ and the effective temperature are then obtained

$F_{\text {int }}=L_{\text {int }} 4 \pi R_{\mathrm{p}}^{2}$

$T_{\mathrm{eff}}=\left[\left(F_{\mathrm{abs}}+F_{\mathrm{int}} / \sigma\right]^{1 / 4}\right.$

being $\sigma$ the Stephan-Boltzman constant.

\section{Atmospheric data}

As stated above we assume that the atmospheres are composed of molecular hydrogen. Condensed phases of some compounds are surely present but they are not considered in our dynamical study. Latent heat release from phase changes could be important in controlling some meteorological processes, as for instance occurs in the development of localized large-scale convective storms in the water clouds of Jupiter and Saturn (Sanchez-Lavega \& Gomez 1996b; Sanchez-Lavega et al. 1991, 1996a). We assume here that they do not play a main role in controlling the global atmospheric dynamics. This is also assumed for the heating effect due to the hydrogen ortho to para conversion because of the high effective temperatures expected in the atmospheres of the planets under study. The mean molecular weight is taken to be $\mu=2.2 \mathrm{gr} \mathrm{mol}^{-1}$ and the specific heat $C_{\mathrm{p}}=14000 \mathrm{~J} \mathrm{~kg}^{-1} \mathrm{~K}^{-1}$. The relevant atmospheric parameters for our study are the following.

The "effective pressure" marks the level where the hydrogen optical depth is 1 . It can be derived from the hydrostatic relation using the ideal gas law and the definition of the optical depth in terms of the hydrogen mass absorption coefficient (see Barnet 1990 for details). For a pure hydrogen atmosphere, it is given by

$P_{\mathrm{eff}}^{2}=\frac{2 g R_{\mathrm{g}} T_{\mathrm{eff}}}{<A_{\nu}>/ \rho_{\mathrm{STP}}^{2}}$

Here $R_{\mathrm{g}}$ is the gas constant, $\left\langle A_{\nu}\right\rangle=3 \times$ $10^{-6} \mathrm{~cm}^{-1} \mathrm{am}^{-1}$, is the absorption coefficient, and $\rho_{\mathrm{STP}}$ is the hydrogen density under standard temperature and pressure.

The scale height for an atmosphere under hydrostatic equilibrium is obtained from

$H=R_{\mathrm{g}} T_{\mathrm{eff}} / \mu g$ and consequently the sound speed is given by

$c_{\mathrm{s}}=(\gamma g H)^{1 / 2}$

with $\gamma=1.4$.

This velocity can be expected to represent an upper limit for the horizontal wind (mass motion) speeds present in the tropospheres. On the other hand, the most intense expected vertical motions should be those related to convection in the troposphere. They can be characterized by means of the mixing length theory (Spiegel 1971; Stone 1976), so the vertical velocities within the convective layer are given by

$w=\left[\frac{R_{\mathrm{g}}}{\mu} \frac{F_{\text {int }}}{\rho C_{\mathrm{p}}}\right]^{1 / 3}$

and taking the mixing - length scale to be the atmospheric scale height, we can define a local convective time as

$t_{\text {conv }}=H / w$.

Other characteristic time scales in the atmosphere are the radiative time constant $\left(t_{\mathrm{rad}}\right)$ and the dynamical time constant $\left(t_{\mathrm{dyn}}\right)$. The radiative time constant gives a measure of the inertia of the atmosphere to heating or cooling (Chamberlain \& Hunten 1987) and is calculated at the level $P_{\text {eff }}$,

$t_{\mathrm{rad}}=\frac{C_{\mathrm{p}} P_{\mathrm{eff}}}{\sigma g T_{\mathrm{eff}}^{3}}$

Taking the planetary radius $R_{\mathrm{p}}$ as a representative scale for the length of the horizontal motions and adopting as an upper limit for the horizontal wind speed the sound velocity $c_{\mathrm{s}}$, we get the dynamical time scale

$t_{\text {dyn }}=R_{\mathrm{p}} / c_{\mathrm{s}}$.

Table 4 gives the characteristic values for these parameters using the data in the previous tables. Most remarkable in this table is the large scale-height found in the atmospheres of CEGP and the shortest one in the brown dwarf Gl229B. Moreover, note the very short radiative time constants in the objects at high temperatures. Except for these objects, we note that in general, $t_{\mathrm{dyn}}<<t_{\text {rad }}$ and $t_{\text {con }}<<t_{\text {rad }}$. 
Table 4. Atmospheric data.

\begin{tabular}{cccccccc}
\hline Object & $\begin{array}{c}P_{\text {eff }} \\
(\mathrm{bar})\end{array}$ & $\begin{array}{c}H \\
(\mathrm{~km})\end{array}$ & $\begin{array}{c}c_{\mathrm{s}} \\
\left(\mathrm{m} \mathrm{s}^{-1}\right)\end{array}$ & $\begin{array}{c}w \\
\left(\mathrm{~m} \mathrm{~s}^{-1}\right)\end{array}$ & $\begin{array}{c}t_{\text {conv }} \\
(\mathrm{hr})\end{array}$ & $t_{\text {rad }}$ & $\begin{array}{c}t_{\text {dyn }} \\
(\mathrm{hr})\end{array}$ \\
\hline 51 Peg B & 0.55 & 407 & 2555 & 2 & 150 & 9 days & 10 \\
$\tau$ Boo B & 2.5 & 40 & 950 & 10 & 2 & 0.8 days & 25 \\
HD 209458B & 0.51 & 470 & 2525 & 2 & 200 & 9 days & 11 \\
$v$ And C & 0.7 & 28 & 463 & 3 & 6 & 51 days & 45 \\
$v$ And D & 1 & 6 & 368 & 4 & 1 & 105 days & 59 \\
47 UMa B & 0.7 & 7.5 & 330 & 3 & 2 & 197 days & 66 \\
E Eri B & 0.3 & 23 & 298 & 3 & 6 & 1.7 years & 73 \\
G1 229B & 3.5 & 4 & 2100 & 37 & 0.05 & 2 days & 10 \\
Jupiter & 0.34 & 19 & 800 & 1 & 14 & 6.3 years & 25 \\
\hline
\end{tabular}

\section{Similarity parameters}

We now present a comparative view of the dynamical state of the atmospheres of these bodies through a "similarity analysis" using some basic non-dimensional numbers that characterize the dynamical state of the atmosphere (see e.g. Golitsyn 1979). The "energy balance" $E$ gives the strength of the internal heat source against the star flux (insolation)

$E=\left(F_{\text {abs }}+F_{\text {int }}\right) / F_{\text {abs }}=\left(T_{\text {eff }} / T_{\text {eq }}\right)^{4}$.

When $E>4 / \pi \sim 1.27$, it is expected that the internal heat source play an important role in controlling dynamics (Ingersoll \& Porco 1978).

To assess the importance of the planet rotation rate (Coriolis force) in shaping the atmospheric motions, we use the Rossby number, i.e. the ratio of the rotational period over the dynamical time scale, so according to Eq. (13) we have

$R o=t_{\mathrm{rot}} / t_{\mathrm{dyn}} \sim c_{\mathrm{s}} / R_{\mathrm{p}} \Omega$.

The Coriolis forces play a role in controlling the motions as long as $R o<1$, as occurs in the Earth, Mars and the giant planets.

The action of rotation on vertical motions (buoyancy) can be quantified by a vertical Rossby number

$R o_{v}=\frac{t_{\mathrm{rot}}}{t_{\mathrm{conv}}}=\frac{w}{\Omega H}$.

Thus, when $R o_{v}<1$, the planetary rotation has a notorious effect, deflecting the motion of the ascending parcels.

The Golitsyn number Go characterizes the thermal inertia of the atmosphere or its degree of diabaticity (Golitsyn 1979, 1984; Chamberlain \& Hunten 1987), i.e. the importance of the heating or cooling of the atmosphere by radiation against dynamics and is given by the ratio

$G o=t_{\mathrm{dyn}} / t_{\mathrm{rad}}$.

If $G o<<1$ the atmosphere would be far from radiative equilibrium and its thermal regime is governed mainly by dynamics, leading to small temperature contrasts (motions tend to redistribute heat and to suppress temperature gradients efficiently). If $G o \geq 1$ the global circulation requires a long period to transport heat, the atmosphere is close to the state of local radiative balance, and the dynamics are tuned to this temperature regime.

According to the values of the similarity parameters (Table 5), it becomes evident that the atmospheric dynamics of the objects under consideration can be divided in at least three main categories that encompass Sudarsky et al. (2000) classes:

(A) Synchronized close EGP (CEGP or "roasters" "Hot Jupiters", albedo classes IV and V). Their dynamics is controlled by the strong stellar radiation $(E=1$, $T_{\text {eff }}>900 \mathrm{~K}$ ) and the Coriolis forces have a small influence in shaping the horizontal horizontal motions $\left(R_{0}>1\right)$. The radiative time constant is very short ( $\sim$ days) and since $G o<1$, the diabatic heat transport by atmospheric motions should have an important role in establishing the thermal state of the atmosphere. One exception is the planet $\tau$ Boo B that has Go $\sim 1$ and so its upper troposphere should be dominated by radiative effects alone. It is at deeper tropospheric levels where convection in this object must be very intense (see below).

(B) Intermediate EGP with dynamical properties similar to those of Jupiter (albedo classes I, II and III). The tropospheric dynamics is controlled most probably by both the internal and external heat source (they have moderate values of $E \sim 2-9$ ) with the rotation effects being very important in shaping the motions $(R o \leq 1)$. The diabatic heat transport should establish small horizontal temperature gradients and the atmosphere should be locally far from radiative equilibrium ( $G o<<1$ ).

(C) Cold brown dwarfs, with the dynamics strongly controlled by the internal heat source $(E>>1)$. Intense vertical motions are expected due to convective heat transport from the interior.

Note that there are many planets where $R o_{v}>1$ if one assumes that groups $\mathrm{B}$ and $\mathrm{C}$ have typically $t_{\text {rot }}=$ $10 \mathrm{hr}$, and that the synchronous rotation time characterizes group A. Convective motions should play a more important role in these objects than in Jupiter. It is somewhat surprising to find in particular a high value $R o_{v} \sim 35$ in two very different objects, $\tau$ Boo B and Gl 229B. The reason is that $\tau$ Boo $\mathrm{B}$ is expected to have an intense internal energy source deeper in its atmosphere (at the level where radiation does not penetrate) and thus the vertical motions should dominate the dynamics. 
Table 5. Similarity parameters.

\begin{tabular}{|c|c|c|c|c|c|c|}
\hline Object & $E$ & Ro & $R o_{v}$ & Go & $t_{\mathrm{orb}} / t_{\mathrm{rad}}$ & $t_{\mathrm{rot}} / t_{\mathrm{rad}}$ \\
\hline 51 Peg B & 1 & 10 & 0.6 & 0.05 & \multirow{3}{*}{ - } & \multirow{3}{*}{ - } \\
\hline$\tau$ Boo B & 1 & 3 & 35 & 1.2 & & \\
\hline HD 209458B & 1 & 8 & 0.4 & 0.05 & & \\
\hline$v$ And $\mathrm{C}$ & 1 & $>0.05$ & 2 & 0.04 & \multirow{5}{*}{$3-13$} & \multirow{5}{*}{$\begin{array}{c}5 \times 10^{-2}- \\
2 \times 10^{-4}\end{array}$} \\
\hline$v$ And D & 3.4 & $>0.02$ & 13 & 0.02 & & \\
\hline 47 UMa B & 2.2 & $>0.02$ & 6 & 0.01 & & \\
\hline$\varepsilon$ Eri B & 9 & $>0.04$ & 2 & 0.005 & & \\
\hline G1 229B & $10^{7}$ & $>0.2$ & 33 & 0.2 & & \\
\hline Jupiter & 1.67 & 0.4 & 0.7 & $7.2 \times 10^{-4}$ & 5.2 & $5 \times 10^{-4}$ \\
\hline
\end{tabular}

Finally, two other ratios permit one to asses the importance of the annual, i.e. seasonal changes, $\left(t_{\text {orb }} / t_{\text {rad }}\right)$, and the diurnal radiative variations $\left(t_{\mathrm{rot}} / t_{\mathrm{rad}}\right)$ in the dynamical state of the atmosphere. They are valuable as long as $E \sim 1$ (i.e. when the stellar insolation is important) and when the planet is not synchronized. From the data in Table 5 we can see that this applies to our group B planets, where $t_{\text {orb }} / t_{\text {rad }} \sim 3-13$ and $t_{\text {rot }} / t_{\text {rad }} \sim 0.05-0.0002$. This indicates that the tropospheres of these planets would have slow adjustment times to the radiative forcing during the diurnal cycle but could have noticeable adjustments during their annual cycle.

\section{Discussion and conclusions}

We have presented a first order estimation, through a similarity theory, of the dynamical state of the atmospheres of giant extrasolar planets and cold brown dwarfs. A broad but distinctive dynamical classification scheme emerges from this analysis. Three main families result, each one represented by archetype planets whose properties are at present best measured or constrained. They follow a dynamical classification where $E$ is the main parameter. The other constraint to dynamics is the angular rotation that tends to make the motions two-dimensional or quasihorizontal as rotation increases.

For group A objects (slowly rotating but strongly irradiated planets), the motions should be driven by the intense winds blowing between the heated and cooled hemispheres. Objects with increasing $E$ but under rapid rotation should develop two dimensional motions in geostrophic balance because of the increasing intensity of Coriolis forces. This is most plausible for group B planets. Based on our experience with Jupiter and Saturn, a system of zonal (East-West) jets varying their intensity with latitude (Limaye 1986; Sanchez-Lavega et al. 2000) could be the main dynamical mode there. Finally, for those cases where $E$ is enormous, as in group C, three-dimensional convective motions driven by the internal energy source should be the main dynamical mode. Even under rapid rotation, the vertical motions are so vigorous in these objects that rotation cannot regulate the visible motion field.

All this tells us that dynamics could play a significant role in establishing the thermal state of the atmosphere of extrasolar planets. Thus, "pure radiative" equilibrium models, such as those used to infer the cloud vertical structure and albedos, should be handled with care. Future advances in atmospheric circulation studies will require the knowledge of the rotation period of these bodies. In the meantime, the CEGP family, whose rotation state and other properties are well constrained (in particular the planet HD 209458 B), should be the main objective of detailed dynamical modeling (Guillot \& Showman 2001).

Acknowledgements. This work was supported by Spanish MCYT research grant PNAYA2000-0932.

\section{References}

Barnet, C. D. 1990, Saturn's Seasonal Winds and Temperature: The Effect of the Ring System on the Troposphere and Stratosphere, Ph.D. Thesis, New Mexico State University, Las Cruces

Bodenheimer, P., Lin, D. N. C., \& Mardling, R. 2001, ApJ, submitted

Brown, T. M., Charbonneau, D., Gilliland, et al. 2001, ApJ, submitted, preprint [astro-ph/0101336]

Burrows, A., Marley, M., Hubbard, W. B., et al. 1997, ApJ, 491,856

Burrows, A., Guillot, T., Hubbard, W. B., et al. 2000, ApJ, 534, L97

Cameron, A. C., Horne, K., Penny, A., \& James, D. 1999, Nature, 402, 751

Cameron, A. C., Horne, K., Penny, A., et al. 2000, in Planetary Systems in the Universe: Observation, Formation and Evolution, ed. A. J. Penny, P. Artymowicz, A.-M. Lagrange, \& S. S. Russell, ASP Conf. Ser., in press

Chamberlain, J. W., \& Hunten, D. M. 1987, Theory of Planetary Atmospheres (Academic Press, San Diego)

Charbonneau, D., Noyes, R. W., \& Korzennik, S. G. 1999, ApJ, $522, \mathrm{~L} 145$

Charbonneau, D., Brown, T. M., Latham, D., \& Mayor, M. 2000a, ApJ, 529, L45

François, P., Spite, M., Guillet, D., et al. 1996, A\&A, 310, L13

Gierasch, P., Goody, R. M., \& Stone, P. 1970, Geophys. Fluid Dyn., 1, 1

Gierasch, P. J. 1976, Icarus, 29, 445

Gierasch, P. J., Goody, R. M., Young, R. E., et al. 1999, The General Circulation of the Venus Atmosphere: An Assessment, ed. S. W. Bougher, D. M. Hunten, \& R. J. Phillips, Venus II (Univ. Arizona Press, Tucson) 
Golitsyn, G. S. 1979, Icarus, 38, 333

Golitsyn, G. S. 1984, Icarus, 60, 289

Gonzalez, G. 1998, A\&A, 334, 221

Guillot, T. 1999, Science, 286, 72

Guillot, T., \& Showman, A. P. 2001, A\&A, in press

Han, I., Black, D. C., \& Gatewood, G. 2000, ApJ, submitted

Hatzes, A. P., Cochran, W. D., McArthur, B., et al. 2000, ApJ, in press

Henry, G., Baliunas, S., Donahue, R., et al. 1997, ApJ, 474, 503

Henry, G. W., Marcy, G. W., Butler, R. P., \& Vogt, S. S. 2000a, ApJ, 529, L41

Henry, G., Baliunas, S., Donahue, R., et al. 2000b, ApJ, 531, 415

Hide, R. 1966, Planet. Space Sci., 14, 669

Ingersoll, A. P. 1990, Science, 248, 308

Ingersoll, A. P., \& Porco, C. C. 1978, Icarus, 35, 27

Jha, S., Charbonneau, D., Garnavich, P. M., et al. 2000, ApJ, 540, L45

Leggett, S. K., Toomey, D., Geballe, T., et al. 1999, ApJ, 517, L139

Limaye, S. J. 1986, Icarus, 65, 335

Marcy, G. W., Butler, R. P., Williams, E., et al. 1997, ApJ, 481,926
Marley, M. S., Gelino, C., Stephens, D., et al. 1999, ApJ, 513, 879

Martin, E., Basri, G., Delfosse, X., et al. 1997, A\&A, 327, L29

Mazeh, T., Zucker, S., Dalla Torre, A., et al. 1999, ApJ, 522, L149

Mazeh, T., Naef, D., Torres, G., et al. 2000, ApJ, 532, L55

Perryman, M. A. C., Lindegren, L., Arenouf, B., et al. 1996, A\&A, 310, L21

Sanchez-Lavega, A., Colas, F., Lecacheux, J., et al. 1991, Nature, 353, 397

Sanchez-Lavega, A., Lecacheux, J., Gomez, J. M., et al. 1996, Science, 271, 631

Sanchez-Lavega, A., \& Gomez, J. M. 1996, Icarus, 121, 1

Sanchez-Lavega, A., Rojas, J. F., \& Sada, P. 2000, Icarus, 147, 405

Schneider, J. 2001, Encyclopedia of Extrasolar Planets, http://www. obspm.fr/encycl/encycl.html

Spiegel, E. A. 1971, ARA\&A, 9, 323

Stone, P. H. 1976, The Meteorology of the Jovian Atmosphere, ed. T. Gehrels, Jupiter (Univ. Arizona Press, Tucson)

Sudarsky, D., Burrows, A., \& Pinto, P. 2000, ApJ, 538, 885

Tinney, C. G., \& Reid, I. N. 1998, MNRAS, 301, 1031

Tinney, C. G., \& Tolley, A. J. 1999, MNRAS, 304, 119 